\title{
Gibberellins enhance plant growth and ginsenoside content in Panax ginseng
}

\author{
Chang Pyo Hong $\cdot$ Gwi Yeong Jang $\cdot$ Hojin Ryu
}

Received: 4 September 2021 / Revised: 23 September 2021 / Accepted: 23 September 2021

(c) Korean Society for Plant Biotechnology

\begin{abstract}
The roots of Korean ginseng (Panax ginseng) have a long history of usage as a medicinal drug. Ginsenosides, a group of triterpenioid saponins in ginseng, have been reported to show important pharmacological effects. Many studies have attempted to identify the ginsenoside synthesis pathways of $P$. ginseng and to increase crop productivity. Recent studies have shown that exogenous gibberellin (GA) treatments promote storage root secondary growth by integration of the modulating cambium stem cell homeostasis with a secondary cell wall-related gene network. However, the dynamic regulation of ginsenoside synthesisrelated genes and their contents by external signaling cues has been rarely evaluated. In this study, we confirmed that GA treatment not only enhanced the secondary growth of $P$. ginseng storage roots, but also significantly enriched the terpenoid biosynthesis process in RNA-seq analysis. Consistently, we also found that the expression of most genes involved in the ginsenoside synthesis pathways, including those encoding methylerythritol-4-phosphate (MEP) and mevalonate (MVA), and the saponin content in both leaves and roots was increased by exogenous GA application. These results can be used in future development of biotechnology for ginseng breeding and enhancement of
\end{abstract}

${ }^{\dagger}$ These authors contributed equally to this work as the first authors C. P. Hong ${ }^{\dagger}$

Theragen Bio Co., Ltd, Suwon 16229, Republic of Korea

G. Y. Jang ${ }^{\dagger}$

Department of Herbal Crop Research, NIHHS, RDA, Eumseong 27709, Republic of Korea

H. Ryu $(\varangle)$

Department of Biology, Chungbuk National University, Cheongju 28644, Republic of Korea

e-mail: hjryu96@chungbuk.ac.kr

H. Ryu

Department of Biological Sciences and Biotechnology, Chungbuk National University, Cheongju 28644, Republic of Korea saponin content.

Keywords Panax ginseng, GA, Hormone, Ginsenoside, Terpenoid biosynthesis

\section{Introduction}

Korean ginseng $(P$. ginseng) is a medicinal herb that contains a lot of functional substances such as saponins and is recognized worldwide for its medicinal efficacy (Baeg and So 2013; Hong et al. 2018; Kaneko and Nakanishi 2004). Ginsenoside, a major physiologically active substances in ginseng, is attracting attention for its excellent pharmacological action. It has been reported to have various effects such as anti-cancer, anti-fatigue, and anti-diabetes etc. (Lee and Kim 2014). The recently advanced integration of genomics and analytical chemistry research has increased our understanding of ginsenoside biosynthesis in plants (Bergman et al. 2019; Hong et al. 2018; Zhao et al. 2014). Ginsenosides are composed of several types of triterpenoid saponins. Terpenoids are synthesized from the precursor isopentenyl pyrophosphate (IPP), which is biosynthesized in both cytoplasmic MVA and plastid MEP pathways. (Dubey et al. 2003; Kim et al. 2018; Zhao et al. 2014). The 2,3-oxidosqualene produced via the MVA and MEP pathways, is biosynthesized to ginsenosides via cyclization, hydroxylation, and finally glycosylation (Kim et al. 2018; Zhao et al. 2014). Similar to most plants, the 2,3-oxidosqualene is cyclized into cycloartenol, $\alpha-, \beta$-amyrin or lupeol, to be further converted to phytosterols and pentacyclic triterpenoids in P. ginseng (Benveniste 2004; Kim et al. 2018). Then, the major dammarane-type saponins [protopanaxadiol (PPD)/protopanaxatriol (PPT)] are synthesized by a specific cyclization reaction activated by a set of cytochrome P450 enzymes. It is further glycosylated via several types of UDP-glycosyltransferases (UGTs) to synthesize ginsenosides. Most of 
genes involved in the ginsenoside synthesis pathway have been identified through recent studies (Benveniste 2004; Kim et al. 2018).

The secondary growth driving from cambium stem cells is a major factor for the yield of $P$. ginseng. It has been recently reported that secondary growth of storage root in $P$. ginseng is regulated by brassinosteroid (BR) and gibberellin (GA) (Hwang et al. 2020; Lee et al. 2018). BR signaling activated by prolonged high ambient temperature inhibited the cell division of cambium stem cells in the tap roots of $P$. ginseng by yet unknown mechanisms (Hwang et al. 2020). Our recent study shows that exogenous GA treatments promoted the secondary growth of ginseng storage roots, and the gene network related to secondary cell wall synthesis is critically involved in this process (Hong et al. 2021). The RNA-seq analysis of GA-treated ginseng roots showed that the terms related to secondary cell wall synthesis formed a close network with genes related to cell division, cell elongation, jasmonic acid (JA) and nitrogen assimilation for promoting secondary growth of the ginseng roots (Hong et al. 2021). In addition, it was recently reported that genes related to canonical GA signaling pathway are evolutionary conserved in the genome of $P$. ginseng (Hong et al. 2021; Jo et al. 2017; Kim et al. 2021). These results indicate that hormones are essentially involved in the secondary growth of the storage root of $P$. ginseng.

Recently, as the concept of well-being and consumer demand for eco-friendly agricultural products increase, research on the development of eco-friendly ginseng cultivation technology is being widely conducted. The well-being trend is expected to emerge as one of the big issues in the future ginseng market. With these demands, recent studies of $P$. ginseng have been focused on the mechanism of pharmacological action in animal model systems and manual development for eco-friendly cultivation (Bergman et al. 2019; Lee et al. 2020; Ratan et al. 2021; Wang et al. 2020). In addition, research is underway to measure the change in ginsenoside content of ginseng sprouts caused by various external environmental factors in the greenhouse system (Li et al. 2016; Huang et al. 2013; Rahimi et al. 2016; Zhang et al. 2021). However, genetic and physiological studies related to the increase in the yield of $P$. ginseng and the synthesis of saponin content have been rarely conducted. In this study, we found that the secondary growth and ginsenoside content was increased in GA-treated ginseng roots. It was also consistently confirmed that the expression of genes involved in ginsenoside synthesis through RNA-seq analysis was significantly increased by GA treatment in the ginseng roots. Finally, our results also showed that ex- ogenous GA treatment in roots increased plant growth and saponin content in both roots and leaves.

\section{Materials and Methods}

Plant growth and RNA-seq analysis

One-year-old $P$. ginseng seedlings (Yunpoong, kindly supplied by National Institute of Horticultural and Herbal Science) were grown on the ginseng cultivation soil medium. After two weeks, the developed ginseng seedlings were treated $10 \mu \mathrm{M} \mathrm{GA}_{3}$ and DMSO (mock treatment) once a week for eight weeks with a soaking method. The GA-treated ginseng plants were sampled, and the patterns of shoot primary growth and main root secondary growth were further analyzed.

RNA-Seq reads of DMSO- and GA-treated ginseng roots, of which three replicates have deposited with the accession number SAMN12240097 and SAMN122731128in NCBI Short Read Archive database (Hong et al. 2021), were reanalyzed for this study. The RNA-seq analysis was performed as described in our previous work (Lee et al., 2021). Genes differentially expressed between each replicate of DMSO- and GA-treated root samples were identified using DESeq2 (Love et al. 2014), based on cutoff values of $q$ $<0.05$ and $\mathrm{FC} \geq 1.5$. GO enrichment analysis of DEGs homologous to Arabidopsis genes (TAIR 10 release) was performed using DAVID, with an EASE score cutoff of $<$ 0.01 . Genes enriched under specific GO terms were selected, and their expression patterns were visualized as a heatmap using $\mathrm{MeV}$ (http://mev.tm4.org). Expression levels of genes were shown as Z-score of TPM values. To test if a particular gene set (i.e., Terpenoid biosynthesis proecess) was enriched, GSEA (Subramanian et al. 2005; www.gsea-msigdb. org/gsea/index.jsp) was applied to a background dataset comprising transcripts with TPM $>0.3$, which balances the numbers of false positives and false negatives, in either DMSO- or GA-treated root samples.

Measurement of ginsenoside composition

The dried ginseng roots and leaves were ground using a hammer mill (Micro hammer cutter mill type-3, Culatti AG, Zurich, Switzerland), and these powders were used for analysis. A $50 \mathrm{mg}$ of sample (ginseng roots and leaves powder) was extracted with $2 \mathrm{~mL}$ of $70 \%$ ethanol solution using a sonicator at room temperature for $1 \mathrm{hr}$, and the extraction process was repeated three times. The extract was diluted to 10 -fold dilution with distilled water, loaded 
on to Diaion HP-20 open column (20 mm $\times 10 \mathrm{~mm}$ ), sequentially eluted with distilled water and methanol, and the methanol fraction was used to HPLC analysis of ginsenoside composition.

Ginsenoside standards (Rg1, Re, Rb1, Rg2(S), Rc, Rg2(R), Rb2, Rb3, F1, Rg6, F4, Rk3, Rh4, Rg3(S), Rg3(R), protopanaxatrio, Rk1, Compound K, Rg5, Rh2, and protopanaxadiol) were purchased from Chengdu BiopurifyPhytochemicals Ltd. (Chengdu, Sichuan, China). HPLC-grade water and acetonitrile (ACN) were purchased from J.T. Baker (Phillipsburg, NJ, USA). Diaion HP20 resin was purchased from Sigma Aldrich (USA). All other chemicals used were of reagent grade.

The ginsenoside composition was analyzed using a highperformance liquid chromatography (HPLC) with a UV-visible detector (HPLC system: 1200 series, Agilent technologies, Santa Clara, CA, USA; column: Kinetex XB-C18, $100 \times$ $4.6 \mathrm{~mm}, 2.6 \mu \mathrm{m}$, Phenomenex, Torrance, CA, USA). The mobile phase of the analytical system consisted of ACN (A) and water (B), using the following gradient: $0 \mathrm{~min}$ (18\% A), 0-5 min (18 22\% A), 5-15 min (22 22\% A), 15-19 min (22 35\% A), 19-25 min (35 35\% A), 25-31 $\min (35-50 \% \mathrm{~A}), 31-51 \min (50 \sim 65 \% \mathrm{~A}), 51-55 \min (65$ $\sim 100 \% \mathrm{~A})$, and $55-63 \mathrm{~min}(100 \sim 100 \% \mathrm{~A})$. The flow rate, detection wavelength, and injection volume were set at $1.0 \mathrm{~mL} / \mathrm{min}, 203 \mathrm{~nm}$, and $10 \mu \mathrm{L}$, respectively.

\section{Results and Discussion}

Our previous study showed that exogenous GA treatments greatly enhanced the secondary growth of the tap roots of $P$. ginseng (Hong et al. 2021). To further investigate the physiological effects of GA in plant growth and secondary metabolites in $P$. ginseng, we reanalyzed the phenotypes and RNA-seq data of $\mathrm{GA}_{3}$-treated roots of 1-year-old $P$. ginseng. As previously reported, exogenous application of GA significantly promoted both stem growth and tap root grith of ginseng compared with DMSO treatment (Figure 1A, B and C). GA treatment increased the length of the ginseng stem and the area of the root by about $75 \%$ and $50 \%$, respectively (Fig. 1B and C). To investigate the relationship between plant growth promotion and the accumulation of secondary metabolites, we next conducted that the GO term related to the metabolic process was identified in the previously reported RNA-seq data of GA3-treated ginseng roots (Hong et al. 2021). As present in Figure. 1D, the gene set enrichment analysis (GSEA) of differentially expressed genes (DEGs) revealed that the upregulated genes in GA treatment were significantly enriched under the functional categories of terpenoid biosynthesis process (FDR : 0.0; Fig. 1D and Table 1). Terpenoid is a representative plant secondary metabolite involved in
A

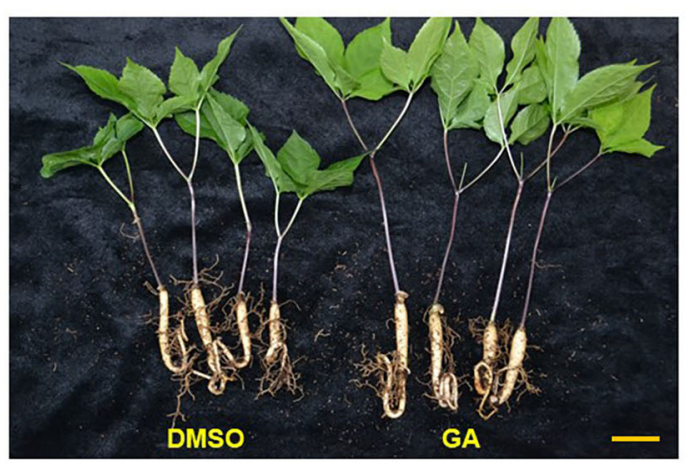

B

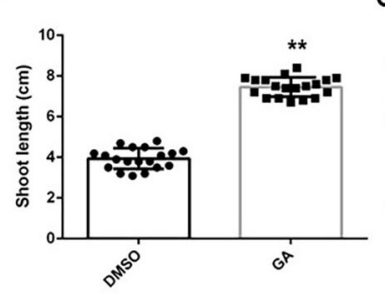

C

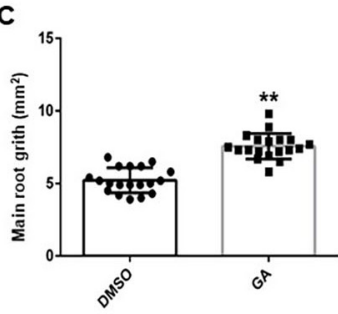

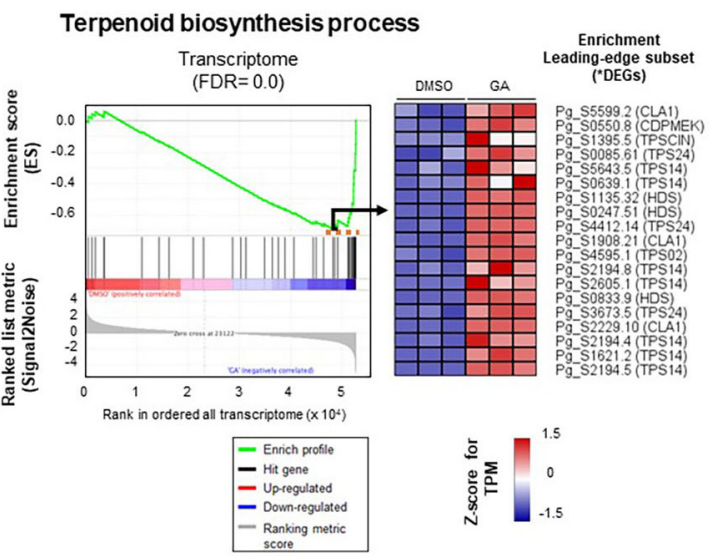

Fig. 1 Exogenous GA treatment affects plant growth and the expression pattern of terpenoid biosynthesis-related genes. (A) GA treatment enhances primary and secondary growth of shoot and storage roots, respectively. One-year-old $P$. ginseng plants were treated with DMSO as a mock control or $10 \mu \mathrm{M} \mathrm{GA} 3$ once a week for 8 weeks. Scale bar $=2 \mathrm{~cm}$. (B, C) Measurements of shoot length (B) and root grith (C). Error bars represent the standard error $(n>18)$. Different lowercase letters indicate statistically significant differences $(P<0.05$; one-way ANOVA, followed by Tukey's multiple range test). (D) Enrichment plot for the terpenoid biosynthesis process and an expression heatmap of 19 genes related to this pathway (FDR $=0.0$ ) were obtained from the RNA-seq data of the roots of $\mathrm{GA}_{3}$-treated $P$. ginseng (Hong et al. 2021) 
Table 1 Expression of genes involved in triterpene saponin biosynthetic pathway in Panax ginseng

\begin{tabular}{|c|c|c|c|c|c|c|c|c|c|}
\hline \multirow[b]{2}{*}{ No } & \multirow{2}{*}{$\begin{array}{c}\text { Gene ID } \\
\text { (P. ginseng) }\end{array}$} & \multirow{2}{*}{$\begin{array}{l}\text { Gene } \\
\text { name }\end{array}$} & \multicolumn{6}{|c|}{ Expression value (TPM) } & \multirow{2}{*}{$\begin{array}{c}\log _{2} F C \\
\text { (GAOMSO) }\end{array}$} \\
\hline & & & DMSO.1 & DMSO-2 & DMSO-3 & GA-1 & GA.2 & GA-3 & \\
\hline 1 & $\begin{array}{ll}g_{2} .56240 .3 \\
\end{array}$ & AACT & 2079.14 & 2019.95 & 2100.83 & 6691.74 & \begin{tabular}{|l|}
6598.55 \\
\end{tabular} & 6549.87 & 1.68 \\
\hline 2 & Pg_S0022.46 & AACT & 922.51 & 860.03 & 939.15 & 948.53 & 968.36 & 998.86 & 0.10 \\
\hline 3 & \begin{tabular}{|l|}
$P_{2} S 1068.4$ \\
\end{tabular} & AACT & 1285.50 & 1406.31 & 1450.38 & 1473.29 & 1419.25 & 1410.31 & 0.05 \\
\hline 4 & $\begin{array}{ll}g_{-} S 6647.3 \\
\end{array}$ & HMGS & 1140.52 & 1130.40 & 1225.58 & 4606.23 & 4761.72 & 4585.54 & 2.00 \\
\hline 5 & \begin{tabular}{|l|}
$P_{2}{ }_{2} 68896.2$ \\
\end{tabular} & HMGS & 2966.21 & 3000.86 & 3085.63 & 11007.48 & 11475.46 & 11253.62 & 1.90 \\
\hline 6 & Pg_S0849.33 & HMGS & 1135.15 & 1350.02 & 1171.94 & 1676.32 & 1694.64 & 1924.63 & 0.53 \\
\hline 7 & $\begin{array}{ll}g_{-} S 4594.4 \\
\end{array}$ & HMGS & 13.96 & 4.61 & 4.56 & 6.25 & 16.20 & 9.02 & 0.44 \\
\hline 8 & \begin{tabular}{|l|}
$P_{2}$ S2776.2 \\
\end{tabular} & HMGS & 4.30 & 7.38 & 4.56 & 1.04 & 4.50 & 4.51 & .0 .69 \\
\hline 9 & $\begin{array}{l}\text { P_S0913.16 } \\
\end{array}$ & HMGR & 1929.86 & 1899.99 & 1927.38 & 5920.22 & 5742.68 & 5508.61 & 1.58 \\
\hline 10 & \begin{tabular}{|l|} 
Pg_S3959.3 \\
\end{tabular} & HMGR & 11.81 & 1.85 & 3.42 & 14.58 & 10.80 & 10.83 & 1.08 \\
\hline 11 & Pg_S0126.10 & HMGR & 2836.27 & 2798.77 & 2916.74 & 5403.79 & 5284.60 & 5424.69 & 0.91 \\
\hline 12 & Pg_S1295.30 & HMGR & 0.00 & 2.77 & 2.28 & 1.04 & 7.20 & 0.90 & 0.86 \\
\hline 13 & \begin{tabular}{|l|}
$P g_{-} S 6083.2$ \\
\end{tabular} & HMGR & \begin{tabular}{|l|l|}
1475.59 \\
\end{tabular} & 1350.94 & 1418.43 & 1914.75 & 1990.72 & \begin{tabular}{|l|}
2033.81 \\
\end{tabular} & 0.48 \\
\hline 14 & \begin{tabular}{|l|}
$P_{-} S 6137.4$ \\
\end{tabular} & HMGR & 616.44 & 613.64 & 631.05 & 599.73 & 592.18 & \begin{tabular}{|l|l|}
641.54 \\
\end{tabular} & -0.02 \\
\hline 15 & $\begin{array}{l}P_{2} S 1114.3 \\
\end{array}$ & MVK & 255.60 & 242.69 & 257.90 & 681.98 & 632.68 & 700.19 & 1.41 \\
\hline 16 & $\begin{array}{l}P_{2} \text { S0573.2 } \\
\end{array}$ & MVK & 417.76 & 528.75 & 492.97 & 1225.48 & 1248.25 & & 1.36 \\
\hline 17 & \begin{tabular}{|l|}
$P_{2} \mathrm{~S} 3321.6$ \\
\end{tabular} & PMK & 321.11 & 390.33 & 411.95 & 724.67 & 698.37 & 671.32 & 0.90 \\
\hline 18 & $\begin{array}{l}P_{2} S 5751.1 \\
\end{array}$ & PIMK & 413.47 & 382.03 & 402.82 & 587.23 & 496.78 & & 0.44 \\
\hline 19 & $\mathrm{Pg}_{\mathrm{g}} \mathrm{S} 3098.25$ & PMK & 356.55 & 309.13 & 313.81 & 314.44 & 305.99 & 362.73 & 0.01 \\
\hline 20 & $\mathrm{Pg}_{\mathrm{g}} \mathrm{S2636.15}$ & PMK & 137.46 & 171.64 & 155.19 & 120.78 & 145.79 & 124.52 & .0 .25 \\
\hline 21 & \begin{tabular}{|c|}
$P_{g} S 1430.1$ \\
\end{tabular} & MVD & 3928.46 & 3805.52 & 3974.57 & 12257.96 & 12343.03 & 12119.83 & 1.65 \\
\hline 22 & $\begin{array}{l}P_{2} S 3074.4 \\
\end{array}$ & MVD & 410.24 & 350.65 & 406.24 & 367.54 & 358.19 & 324.83 & -0.15 \\
\hline 23 & \begin{tabular}{|l|}
$P_{2}$ S7337.2 \\
\end{tabular} & $|D|$ & 1191.00 & 1171.00 & 1286.06 & 2131.32 & 2129.32 & 2169.15 & 0.82 \\
\hline 24 & $P_{g} S 1168.2$ & 101 & 1048.16 & 987.37 & 991.65 & 1466.00 & 1518.24 & 1474.38 & 0.56 \\
\hline 25 & \begin{tabular}{|l|}
$P g \_S 6728.1$ \\
\end{tabular} & 101 & 0.00 & 0.00 & 1.14 & 0.00 & 0.00 & 0.00 & $\mathrm{NA}$ \\
\hline 26 & Pg_S2229.10 & DXS & 781.83 & 846.18 & 731.47 & 3417.19 & 3478.37 & 3380.06 & 2.12 \\
\hline 27 & $P_{g}$ S 1908.21 & DXS & \begin{tabular}{|l|}
1751.59 \\
\end{tabular} & 1613.93 & 1635.25 & 4762.41 & \begin{tabular}{|l|}
5037.11 \\
\end{tabular} & \begin{tabular}{|l|}
4573.81 \\
\end{tabular} & 1.52 \\
\hline 28 & \begin{tabular}{|l|}
$P g \_S 5599.2$ \\
\end{tabular} & DXS & \begin{tabular}{|l|l|}
615.37 \\
\end{tabular} & 540.75 & 550.03 & 793.39 & 862.17 & 893.29 & 0.58 \\
\hline 29 & \begin{tabular}{|r|}
$g_{-} S 0859.7$ \\
\end{tabular} & DXS & 302.85 & 419.86 & 337.78 & 438.34 & 498.58 & 482.74 & 0.42 \\
\hline 30 & 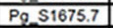 & DXS & 194.38 & 214.08 & 176.88 & 247.80 & 242.09 & 255.35 & 0.35 \\
\hline 31 & $\mathrm{Pg}_{\mathrm{g}} \mathrm{S2533.15}$ & DXS & 218.01 & 176.25 & 241.92 & 214.49 & .99 & 227.38 & 0.13 \\
\hline 32 & $\mathrm{Pg} S 1214.23$ & DXS & 198.68 & 198.40 & 201.98 & 213.44 & 206.99 & 194.90 & 0.04 \\
\hline 33 & $P g$ S3759.10 & DXS & 4.30 & 15.69 & 13.69 & 10.41 & 2.70 & 11.73 & -0.44 \\
\hline 34 & $\mathrm{Pg} S \mathrm{S3733.18}$ & DXR & 651.88 & 757.60 & 715.49 & 2577.99 & 2705.30 & 2512.03 & 1.88 \\
\hline 35 & \begin{tabular}{|l|}
$P g$ \\
\end{tabular} & DXR & 399.50 & 418.02 & 451.89 & 1539.92 & 1601.94 & 1474.38 & 1.86 \\
\hline 36 & 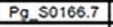 & DXR & 541.26 & 464.16 & 537.47 & 1180.71 & 1239.25 & & 124 \\
\hline 37 & \begin{tabular}{|l|} 
Pg_S4650.8 \\
\end{tabular} & DXR & 2504.42 & 2628.06 & 2486.53 & 5224.70 & 5360.19 & 5087.23 & 1.04 \\
\hline 38 & $P g 05054.32$ & MEP-CT & 237.34 & 241.77 & 230.51 & 841.28 & 818.07 & \begin{tabular}{|l|}
873.44 \\
\end{tabular} & 184 \\
\hline 39 & Pg_S0148.25 & MEP-CT & 99.88 & 105.20 & 99.28 & 265.50 & 222.29 & 247.23 & 1.27 \\
\hline 40 & $\mathrm{Pg}_{\mathrm{g}} \mathrm{SO550.8}$ & \begin{tabular}{|l|} 
CDP-MEKK \\
\end{tabular} & 1023.46 & 991.06 & 947.14 & 1478.49 & 1572.24 & 1450.92 & 0.60 \\
\hline 41 & \begin{tabular}{|l|}
$P_{2} S 2198.2$ \\
\end{tabular} & \begin{tabular}{|l} 
CDP-MEK \\
\end{tabular} & 1363.90 & 1330.64 & 1319.15 & 1901.22 & 1842.23 & 1864.17 & 0.48 \\
\hline 42 & $P_{g}$ S7699.3 & \begin{tabular}{|l|} 
MECDPS \\
\end{tabular} & 1010.57 & 1207.91 & 1116.03 & 6460.60 & 6429.35 & 6585.96 & 2.55 \\
\hline 43 & \begin{tabular}{|l|}
$\mathrm{Pg} S \mathrm{~S} 4369.6$ \\
\end{tabular} & \begin{tabular}{|l} 
MECOPS \\
\end{tabular} & \begin{tabular}{|l|}
916.07 \\
\end{tabular} & 771.44 & 789.66 & 1629.47 & 1646.04 & \begin{tabular}{|l|}
1588.07 \\
\end{tabular} & 0.97 \\
\hline 44 & $\begin{array}{l}P_{2} \text { SO285.3 } \\
\end{array}$ & \begin{tabular}{|l} 
MECDPS \\
\end{tabular} & 395.21 & 460.46 & 486.12 & 573.70 & 543.58 & 634.32 & 0.38 \\
\hline 45 & $P g \_S 1005.15$ & \begin{tabular}{|l|} 
MECDPS \\
\end{tabular} & \begin{tabular}{|l|}
371.58 \\
\end{tabular} & & 346.5 & 409 & 43 & & 0.19 \\
\hline 46 & \begin{tabular}{|l|}
$P_{2} S_{0} 0833.9$ \\
\end{tabular} & \begin{tabular}{|l|} 
HMBPPS \\
\end{tabular} & 2187.61 & 2284.79 & 2325.63 & 7352.90 & \begin{tabular}{|l|l|}
7482.31 \\
\end{tabular} & 7011.86 & 1.68 \\
\hline 47 & $\mathrm{Pg}_{\mathrm{g}} \mathrm{S} 0247.51$ & \begin{tabular}{|l|} 
HMBPPS \\
\end{tabular} & 3012.39 & 3028.54 & 2924.73 & 6905.19 & \begin{tabular}{|l|}
7092.63 \\
\end{tabular} & \begin{tabular}{|l|}
6935.16 \\
\end{tabular} & 1.22 \\
\hline 48 & $P_{g} S 1135.32$ & \begin{tabular}{|l|} 
HMBPPS \\
\end{tabular} & 5310.62 & 5497.89 & 5387.29 & 11587.43 & 11183.87 & 11665.97 & 1.09 \\
\hline 49 & \begin{tabular}{|l|}
$P_{\perp}$ S5991.6 \\
\end{tabular} & \begin{tabular}{|l|} 
HMBPPS \\
\end{tabular} & 316.81 & 296.21 & 279.58 & 279.04 & 257.39 & 238.21 & .0 .20 \\
\hline 50 & \begin{tabular}{|l|}
$g_{2} S 4604.8$ \\
\end{tabular} & \begin{tabular}{|l|} 
HMBPPR \\
\end{tabular} & 4738.21 & 4512.36 & 4609.04 & 8166.07 & 8206.79 & 8062.14 & 0.82 \\
\hline 51 & $P_{g} S 4375.10$ & \begin{tabular}{|l|} 
HMBPPR \\
\end{tabular} & 11.81 & 17.53 & 18.26 & 24.99 & 34.20 & 17.14 & 0.68 \\
\hline 52 & $P g$ S4375.11 & \begin{tabular}{|l|} 
HMBPPR \\
\end{tabular} & 6485.50 & 6476.95 & 6420.0 & 7437.24 & 7440.92 & 7307.81 & 0.19 \\
\hline 53 & \begin{tabular}{|r|}
$P_{2} S_{0} 4447.5$ \\
\end{tabular} & \begin{tabular}{|l|} 
HMBPPR \\
\end{tabular} & 218.01 & 227.93 & 187.15 & 193.66 & 197.99 & 186.78 & -0.13 \\
\hline 54 & $\mathrm{Pg}_{\mathrm{g}} \mathrm{S} 3713.7$ & HMBPPR & 2234.86 & 2272.79 & 2392.96 & \begin{tabular}{|l|}
2013.67 \\
\end{tabular} & \begin{tabular}{|l|}
2168.92 \\
\end{tabular} & \begin{tabular}{|l|}
2059.07 \\
\end{tabular} & -0.14 \\
\hline
\end{tabular}

the synthesis of critical for substances in medicine uses (Bergman et al. 2019; Lee and Kim 2014; Benveniste 2004). These results indicate that GA treatment has a great influence on the promotion of plant growth and the synthesis pathway of secondary metabolites of $P$. ginseng.

Since the synthesis of terpenoids is used as a precursor for the synthesis of ginsenoside in ginseng, we next investigated the expression patterns of genes involved in saponin biosynthetic pathways (Bergman et al. 2019; Kim et al. 2018). It was confirmed that most of the expression of AACT (Acetyl-CoA C-acetyltransferase), HMGS (Hydroxymethylglutaryl-CoA synthase), HMGR (Hydroxymethylglutaryl-CoA reductase), MVK (Mevalonate kinase) and PMK (Phosphomevalonate kinase) genes involved in the MVA pathway occurring in the cytoplasm was increased by GA treatment (Fig. 2A). In addition, we found that the expression of most genes involved in another MEP (occurred in plastid) pathway for IPP synthesis was highly maintained by GA treatment (Fig. 2A). IPP synthesized by either the MEP or

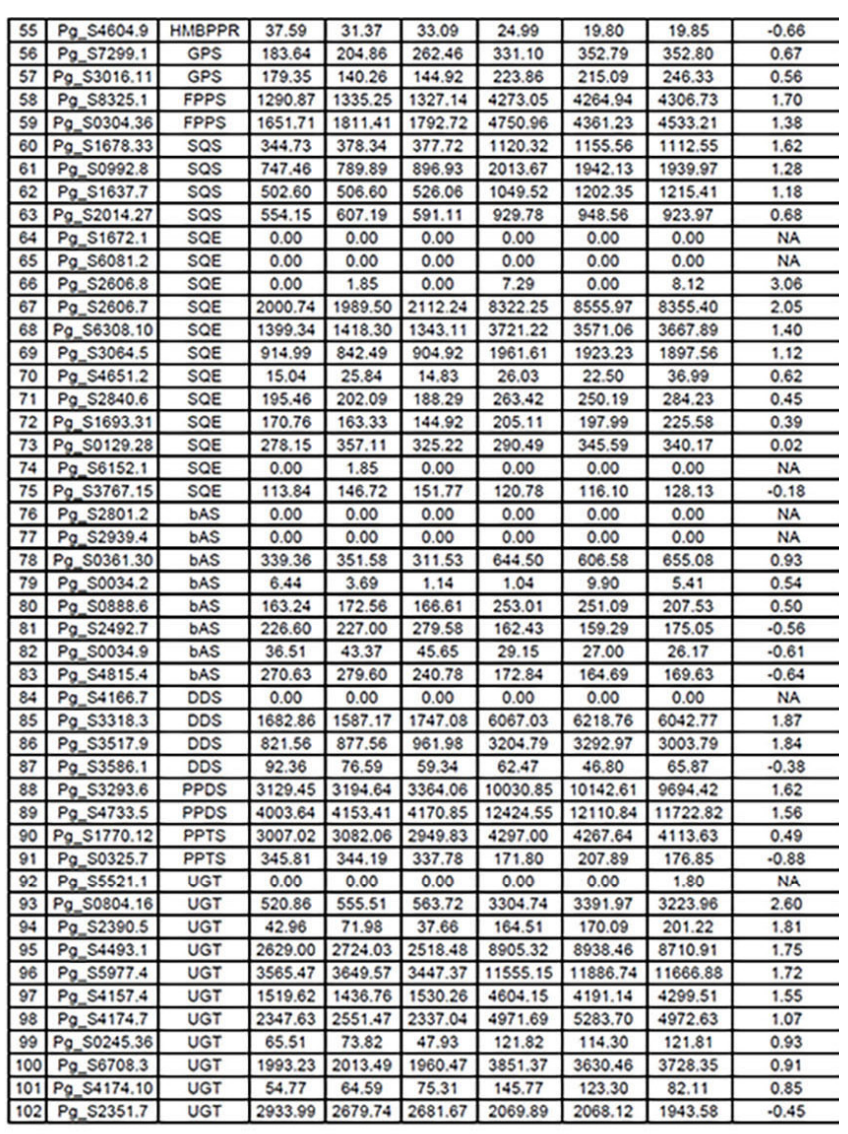

MVA pathways is converted to triterpene and then synthesized to 2,3-oxidosqualene by several SQEs (Squalene epoxidase). Twelve $S Q E$ genes are known in the genome of ginseng, and the expression of $8 S Q E$ genes was increased by GA treatment (Fig. 2B). The 2,3-oxidosqualene was then cyclized to $\beta$-amyrin by the $b$-AS (Squalene cyclase) family, but only 3 genes out of a total of 8 gene families were upregulated in GA-treated ginseng roots. However, the expression of genes involved in the synthesis of dammaranediol, a cyclic compound additionally synthesized by 2,3-oxidosqualene, and the synthesis of major dammarane-type sapogenin [protopanaxadiol (PPD)/protopanaxatriol (PPT)] were mostly upregulated by exogenous $\mathrm{GA}_{3}$ (Fig. 2B). Finally, we confirmed that most UDP-glycosyltransferases that finally synthesize ginsenoside by further glycosylation of these precursors was also highly expressed in the GA-treated ginseng (Fig. 2B). Taken together, these results suggest that the terpenoid synthesis pathway increased by GA treatment would positively control the synthesis of 


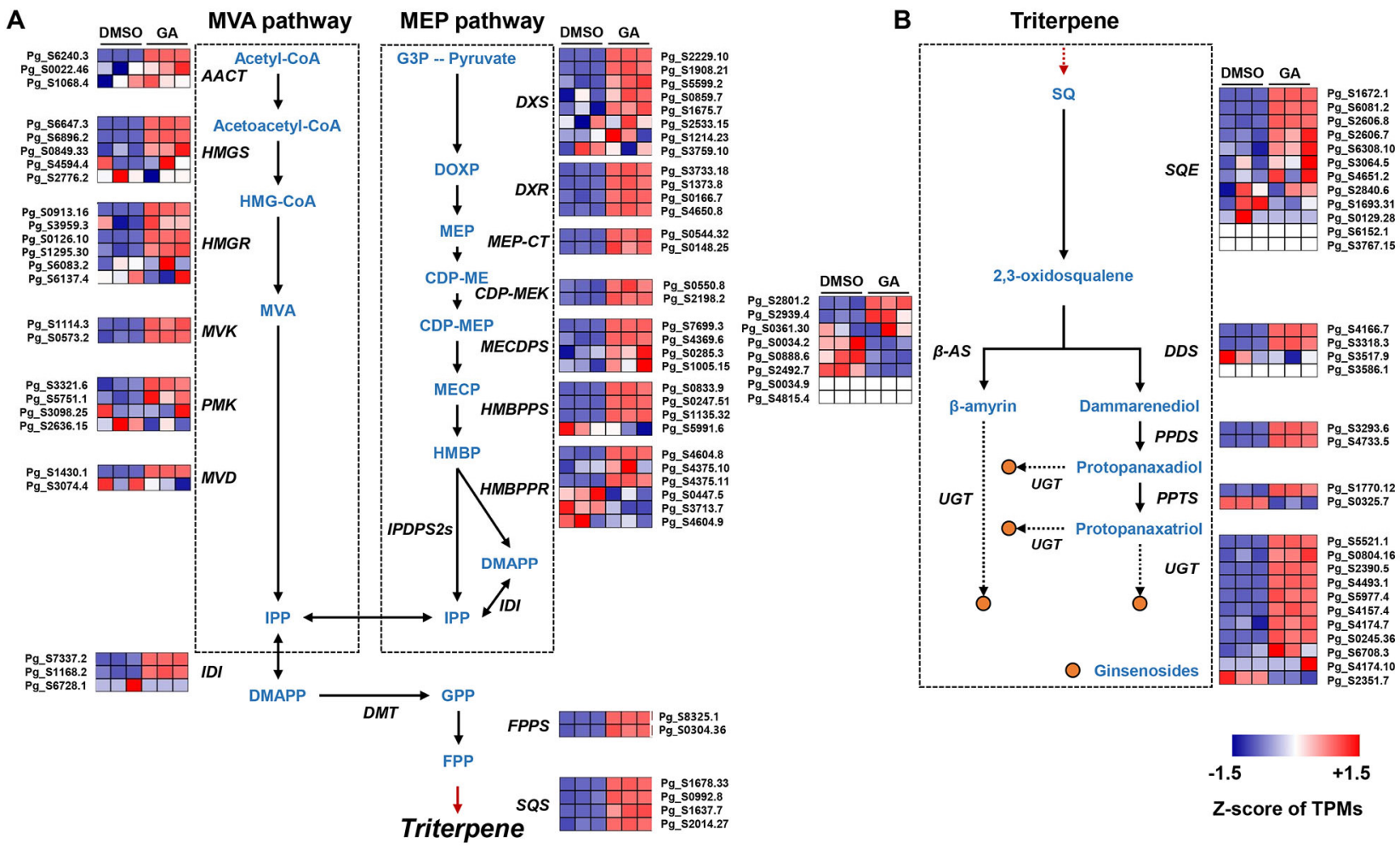

Fig. 2 Transcriptome analysis of the saponin biosynthetic pathways of GA-treated ginseng roots. The ginsenoside biosynthetic pathway and expression levels of its related genes in $P$. ginseng are presented. The expression heatmaps of genes involved in ginsenoside biosynthetic pathways (A; MEP and MVA pathways, B; downstream pathway) were obtained from the RNA-seq data of the roots of $\mathrm{GA}_{3}$-treated P. ginseng (Hong et al. 2021)

various ginsenosides.

We next measure the content of various types of ginsenosides in GA-treated ginseng plants. Typical chromatograms of ginsenosides for $P$. ginseng roots and leaves were initially measured (Fig. 3). Thereafter, the contents of each ginsenoside were comparatively analyzed in the leaves and roots. As shown in Figure. 4, the ginsenoside contents were generally measured to be high in the $\mathrm{GA}_{3}$-treated ginseng samples. In particular, the ginsenoside contents were higher in leave samples than in roots. $\mathrm{Re}$ and $\mathrm{Rb} 2$ types were measured to be high in leaves, and most ginsenosides except for F1 and $\operatorname{Rg} 3(\mathrm{R})$ were increased by about $10 \%$ in GA3-treated ginseng root samples (Fig. 4). However, the content of $\mathrm{Rb} 3$ did not show a significant difference in both leaves and roots (Fig. 4). Taken together, these results indicate that GA treatment can increase the expression and content of genes involved in the synthesis of ginsenoside in ginseng.

In this study, we confirmed once again that the primary and secondary growth of $P$. ginseng was promoted by exogenous GA treatments. Our recent study demonstrates that genes involved in GA signaling in the ginseng genome are evolutionarily well conserved and functional genomic analysis (Kim et al. 2021; Hong et al. 2021). In addition, terms related to the terpenoid synthesis pathway among DEG whose expression is increased by GA treatment are very significantly related, showing that the expression of genes related to saponin synthesis in ginseng is specifically increased. Several studies have demonstrated that transcription factors activated by JA signaling directly reduced GA synthesis and signaling (Heinrich et al. 2013; Cheng et al. 2009). JA is well known as a representative substance that enhances saponin synthesis in the invitro culture of ginseng. This study describes the effect of short-term GA treatment using one-year-old ginseng seedlings. However, several experimental results suggest that GA would enhance saponin synthesis through direct interaction with JA as well as promoting plant growth. These results can be expected to promote the growth of ginseng seedlings and increase saponin in a short period of time, and furthermore, they can be used as important clues in the breeding of $P$. ginseng.

\section{Conflict of Interest Disclosures}

All Authors have read the manuscript and declared that they have no conflict of interest. 

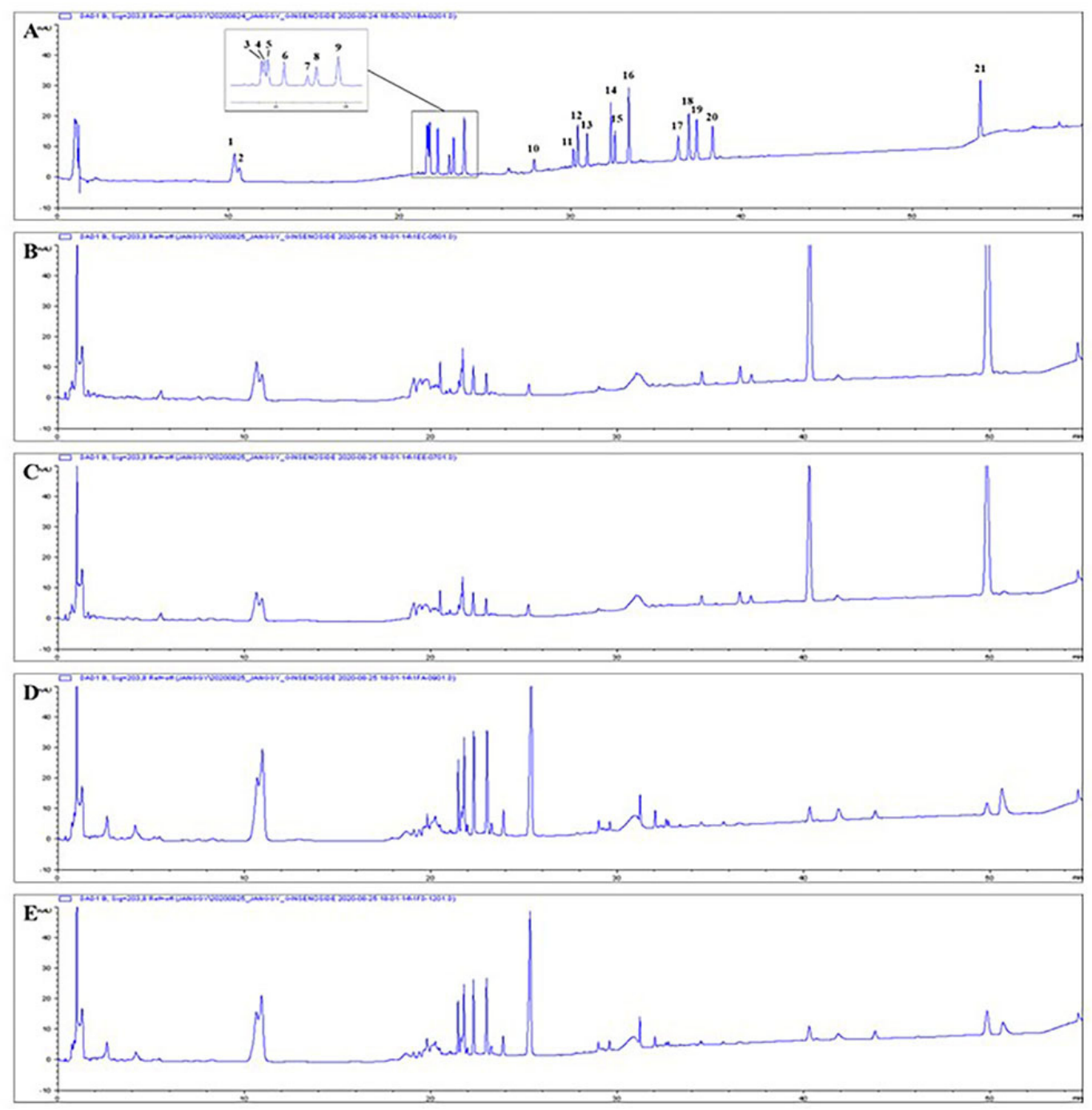

Fig. 3 Typical chromatograms of $P$. ginseng roots and leaves treated with or without $\mathrm{GA}_{3}$. Samples: A, ginsenoside standards; B, roots $\left(\mathrm{GA}_{3}\right)$; C, roots (Con.); D, leaves $\left(\mathrm{GA}_{3}\right)$; E, leaves (Con.). Ginsenoside standards: 1, Rg1; 2, Re; 3, Rb1; 4, Rg2(S); 5, Rc; 6, Rg2(R); 7, Rb2; 8, Rb3; 9, F1; 10, Rg6; 11, F4; 12, Rk3; 13, Rh4; 14, Rg3(S); 15, Rg3(R); 16, protopanaxatriol; 17, Rk1; 18, Compound $\mathrm{K} ; 19$, Rg5; 20, Rh2; 21, protopanaxadiol

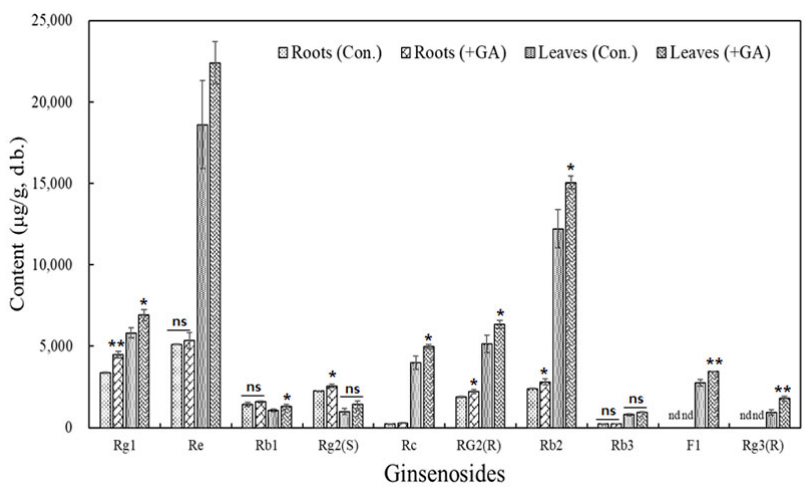

Fig. 4 Ginsenoside content of $P$. ginseng roots and leaves after GA treatment. The ginsenoside content was measured. Error bars indicate $\operatorname{SEM}\left(n=3 ;{ }^{*} p<0.05 ;{ }^{*} p<0.01\right.$; Student's $t$-test). ns: not significant, nd: not detected

\section{Acknowledgments}

This research was supported by Chungbuk National University Korea National University Development Project (2021).

\section{References}

Baeg IH, So SH (2013) The world ginseng market and the ginseng (Korea). J Ginseng Res 37(1):1-7

Benveniste P (2004) Biosynthesis and accumulation of sterols. Annu Rev Plant Biol 55:429-457

Bergman ME, Davis B, Phillips MA (2019) Medically Useful Plant Terpenoids: Biosynthesis, Occurrence, and Mechanism of Action. Molecules 24(21) 
Cheng H, Song S, Xiao L, Soo HM, Cheng Z, Xie D, Peng J (2009) Gibberellin acts through jasmonate to control the expression of MYB21, MYB24, and MYB57 to promote stamen filament growth in Arabidopsis. PLoS Genet 5(3):e1000440

Heinrich M, Hettenhausen C, Lange T, Wunsche H, Fang J, Baldwin IT, Wu J (2013) High levels of jasmonic acid antagonize the biosynthesis of gibberellins and inhibit the growth of Nicotiana attenuata stems. Plant J 73(4):591-606

Hong CP, Kim J, Lee J, Yoo S-i, Bae W, Geem KR, Yu J, Jang I-b, Jo IH, Cho H, Shim D, Ryu H (2021) Gibberellin signaling promotes the secondary growth of storaage roots in Panax ginseng. Int J Mol Sci 22:8694

Hong J, Kim H, Ryu H (2018) Identification of ABSCISIC ACID (ABA) signaling related genes in Panax ginseng. J Plant Biotechnol (45):306-314

Huang C, Qian ZG, Zhong JJ (2013) Enhancement of ginsenoside biosynthesis in cell cultures of Panax ginseng by N,N'dicyclohexylcarbodiimide elicitation. J Biotechnol 165(1):30-36

Hwang H, Lee HY, Ryu H, Cho H (2020) Functional Characterization of BRASSINAZOLE-RESISTANT 1 in Panax Ginseng (PgBZR1) and Brassinosteroid Response during Storage Root Formation. Int J Mol Sci 21(24)

Jo IH, Lee J, Hong CE, Lee DJ, Bae W, Park SG, Ahn YJ, Kim YC, Kim JU, Lee JW, Hyun DY, Rhee SK, Hong CP, Bang KH, Ryu H (2017) Isoform Sequencing Provides a More Comprehensive View of the Panax ginseng Transcriptome. Genes (Basel) 8(9)

Kaneko H, Nakanishi K (2004) Proof of the mysterious efficacy of ginseng: basic and clinical trials: clinical effects of medical ginseng, korean red ginseng: specifically, its anti-stress action for prevention of disease. J Pharmacol Sci 95(2):158-162

Kim NH, Jayakodi M, Lee SC, Choi BS, Jang W, Lee J, Kim HH, Waminal NE, Lakshmanan M, van Nguyen B, Lee YS, Park HS, Koo HJ, Park JY, Perumal S, Joh HJ, Lee H, Kim J, Kim IS, Kim K, Koduru L, Kang KB, Sung SH, Yu Y, Park DS, Choi D, Seo E, Kim S, Kim YC, Hyun DY, Park YI, Kim C, Lee TH, Kim HU, Soh MS, Lee Y, In JG, Kim HS, Kim YM, Yang DC, Wing RA, Lee DY, Paterson AH, Yang TJ (2018) Genome and evolution of the shade-requiring medicinal herb Panax ginseng. Plant Biotechnol J 16(11):1904-1917

Kim J, Shin WR, Kim YH, Shim D, Ryu H (2021) Functional characterization of gibberellin signaling related genes in Panax ginseng. J Plant Biotech 48(3):148-155

Lee $\mathrm{CH}$, Kim JH (2014) A review on the medicinal potentials of ginseng and ginsenosides on cardiovascular diseases. J Ginseng Res 38(3):161-166
Lee DK, Park S, Long NP, Min JE, Kim HM, Yang E, Lee SJ, Lim J, Kwon SW (2020) Research Quality-Based Multivariate Modeling for Comparison of the Pharmacological Effects of Black and Red Ginseng. Nutrients 12(9)

Lee J, Shim D, Moon S, Kim H, Bae W, Kim K, Kim YH, Rhee SK, Hong CP, Hong SY, Lee YJ, Sung J, Ryu H (2018) Genomewide transcriptomic analysis of BR-deficient Micro-Tom reveals correlations between drought stress tolerance and brassinosteroid signaling in tomato. Plant Physiol Biochem 127:553-560

Lee J, Kim H, Park SG, Hwang H, Yoo Si, Bae W, Kim E, Kim J, Lee HY, Heo TY (2021) Brassinosteroid-BZR1/2-WAT1 module determines the high level of auxin signalling in vascular cambium during wood formation. New Phytologist 230(4):1503-1516

Li J, Liu S, Wang J, Li J, Liu D, Li J, Gao W (2016) Fungal elicitors enhance ginsenosides biosynthesis, expression of functional genes as well as signal molecules accumulation in adventitious roots of Panax ginseng C. A. Mey. J Biotechnol 239:106-114

Love MI, Huber W, Anders S (2014) Moderated estimation of fold change and dispersion for RNA-seq data with DESeq2. Genome biology 15(12):1-21

Rahimi S, Kim YJ, Sukweenadhi J, Zhang D, Yang DC (2016) PgLOX6 encoding a lipoxygenase contributes to jasmonic acid biosynthesis and ginsenoside production in Panax ginseng. J Exp Bot 67(21):6007-6019

Ratan ZA, Haidere MF, Hong YH, Park SH, Lee JO, Lee J, Cho JY (2021) Pharmacological potential of ginseng and its major component ginsenosides. J Ginseng Res 45(2): 199-210

Subramanian A, Tamayo P, Mootha VK, Mukherjee S, Ebert BL, Gillette MA, Paulovich A, Pomeroy SL, Golub TR, Lander ES (2005) Gene set enrichment analysis: a knowledge-based approach for interpreting genome-wide expression profiles. Proceedings of the National Academy of Sciences 102 (43):15545-15550

Wang C, Liu J, Deng J, Wang J, Weng W, Chu H, Meng Q (2020) Advances in the chemistry, pharmacological diversity, and metabolism of 20(R)-ginseng saponins. J Ginseng Res 44 (1):14-23

Zhang Y, Jin T, Ryu G, Gao Y (2021) Effects of screw configuration on chemical properties and ginsenosides content of extruded ginseng. Food Sci Nutr 9(1):251-260

Zhao S, Wang L, Liu L, Liang Y, Sun Y, Wu J (2014) Both the mevalonate and the non-mevalonate pathways are involved in ginsenoside biosynthesis. Plant Cell Rep 33(3):393-400 